\title{
Photogrammetric Technique for Center of Gravity Determination
}

\author{
Thomas W. Jones ${ }^{1}$ \\ NASA, Langley Research Center, Hampton, Virginia, 23681 \\ Thomas H. Johnson ${ }^{2}$ \\ NASA, Langley Research Center, Hampton, Virginia, 23681 \\ Dave Shemwell ${ }^{3}$ \\ Systems Planning and Analysis Inc., Alexandria, Virginia, 22311 \\ Christopher M. Shreves ${ }^{4}$ \\ NASA, Wallops Flight Facility, Wallops Island, Virginia, 23337
}

\begin{abstract}
A new measurement technique for determination of the center of gravity (CG) for large scale objects has been demonstrated. The experimental method was conducted as part of an LS-DYNA model validation program for the Max Launch Abort System (MLAS) crew module. The test was conducted on the full scale crew module concept at NASA Langley Research Center. Multi-camera photogrammetry was used to measure the test article in several asymmetric configurations. The objective of these measurements was to provide validation of the CG as computed from the original mechanical design. The methodology, measurement technique, and measurement results are presented.
\end{abstract}

\section{Introduction}

RECENTLY NASA's Orion Project sought test data to anchor the LS-DYNA ${ }^{\circledR}$ analytical model predictions of crew module (CM) water landing loads. These loads are the largest structural mass design driver and were predicted by modeling the physical interaction of the heat shield and side wall structures over a large range of water landing conditions. The range of these predictions meant that the CM structure could be oversized and would be too heavy or undersized and would either present a potentially large development risk late in the program or a safety risk to the crew. To refine this predictions a test of an existing full-scale CM boilerplate was commissioned to serve as a pathfinder for Orion Project water landing analysis. The test program would use only the variables of vertical velocity and entry angle. The water-drop tests were conducted at the Aberdeen Test Center (Aberdeen, Maryland). A graphical representation of a typical water-drop tracking the center of gravity (CG) through water impact is shown in Figure 1.

Frequently as part of a structural validation test program the addition of test components and instrumentation can lead to some degree of uncertainty regrading the known position of the CG of a test article.

To assist the LS-DYNA ${ }^{\circledR}$ model validation for the CM an independent method of determining the crew module center of gravity was investigated. The CG which serves as the origin for all 6 degrees of Freedom (6DOF) measurements was critical for test/model parameter correlation.

\footnotetext{
${ }^{1}$ Aerospace Engineer, RD/Adv Sensing \& Optical Measurements Branch, m/s 493, AIAA Member.

${ }^{2}$ Aerospace Engineer, ED/ Aerospace Systems Engr. Branch, m/s 238, AIAA Member.

${ }^{3}$ Aerospace Engineering Consultant.

${ }^{4}$ Aerospace Engineer, Mechanical Systems Branch, m/s 548
} 


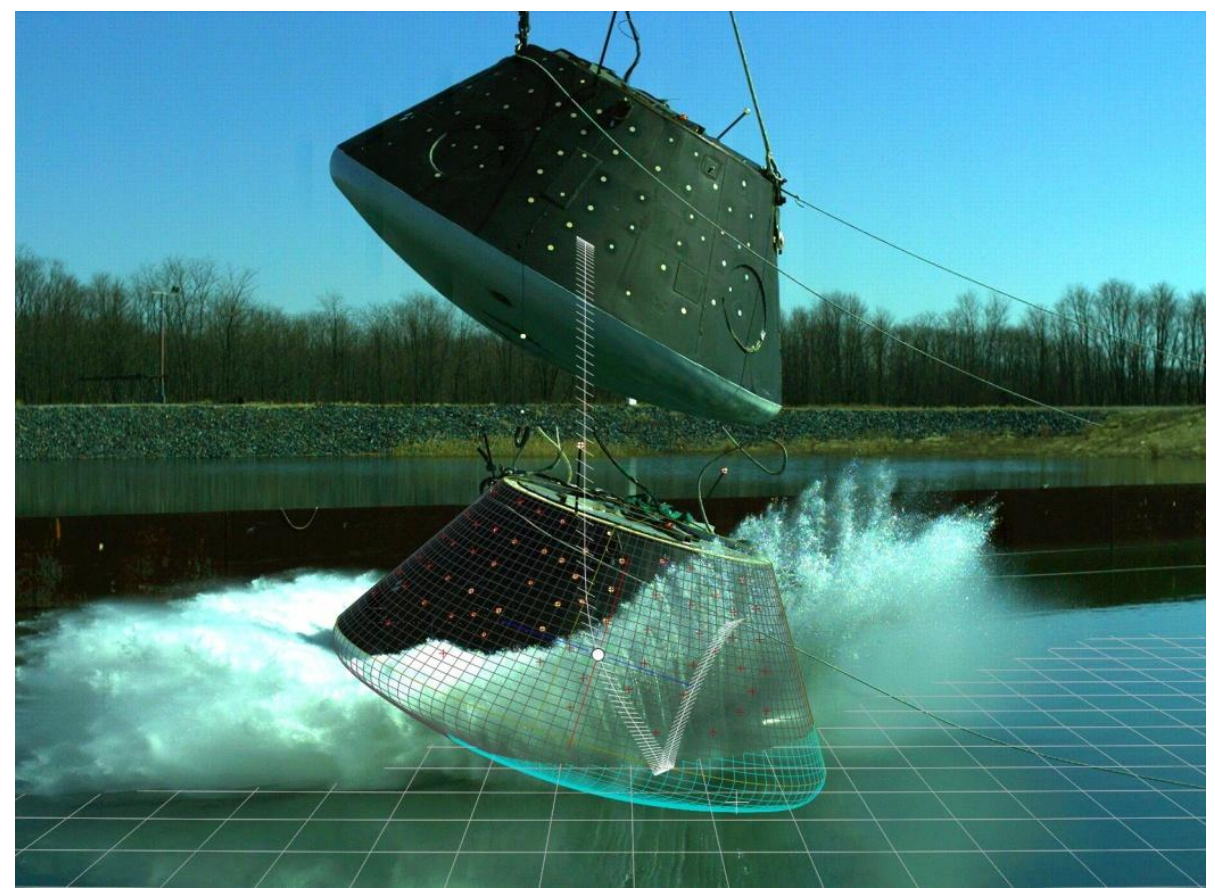

Figure 1. Water drop test of the Orion CM boilerplate with depicted travel of CG.

All flight programs have a basic requirement to measure the mass properties of the payload or flight vehicle to understand the influence on flight dynamics, included among these is the Center of Gravity. To accomplish this NASA has sophisticated laboratory systems such as those at the Mass Properties Measurement Facilities (MPMF) at the Goddard Space Flight Center. One such test platform has a spherical air bearing supporting a circular measurement table (Figure 2). As an example, test loads are supported by the gimbaled spherical bearing under the table. If the test item center of gravity is offset from the table center, the test item, table and spherical bearing will tilt. This angular displacement is reacted by the lower cylindrical bearing with a load cell to measure the moment unbalance. In the center of gravity mode the stabilizing shaft is free to rotate about its axis. The CG measurements are performed by recording the moment (load cell force reading $\times$ distance down from table top) at each of the four quadrant table orientations. This system and method have been used as a standard practice at NASA Goddard Space Flight Center for the last 20 years.

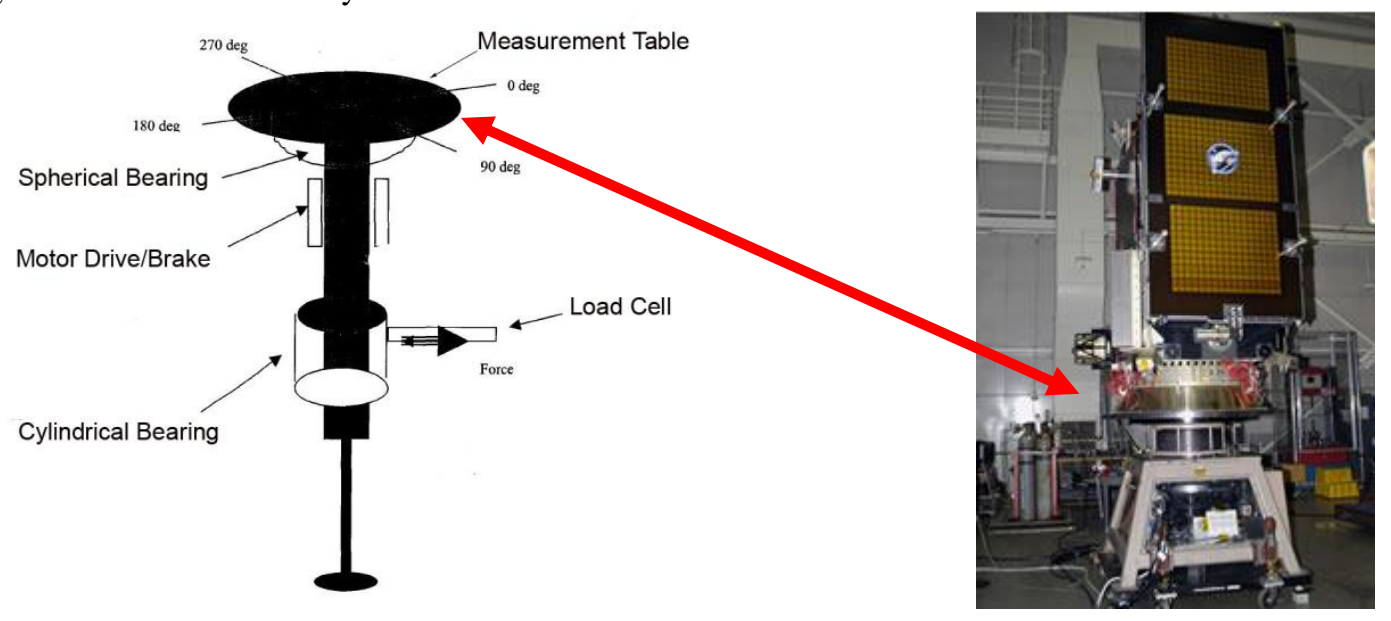

Figure 2. Mass properties test stand at the Goddard Space Flight Center Mass Properties Measurement Facilities (MPMF). 
The extremely large scale of the Orion CM (16.5 ft diameter/17,000 lbs) was in part responsible for the desire to seek a alternative technique to find the CG. The photogrammetric approach for this type of measurement appears to be novel in its method ${ }^{1,2,3,4,5}$. Photographic images are the result of a perspective projection of the three-dimensional (3D) object onto the two dimensional image plane. Consequently, two or more photographic images can be reverseengineered to derive the 3D shape or position of the original object. This process is called photogrammetry, and the solution provides a quantitative relationship between a 3D object and the 2D images acquired by the cameras. While photogrammetry has its roots in the topographic mapping and surveying field, the last decade has seen "closerange" photogrammetric techniques developed to support various industrial and research applications ${ }^{6,7}$. For example, in some areas of aeronautics aeroelastic experimentation (e.g., model deformation and wing twist) photogrammetric measurements has become part of the standard data set. Originally, the techniques were developed for use with still-frame film cameras, but the rapid development of sensor technology has led to the successful migration from film to digital camera and video technology.

Accurate photogrammetric measurements require the photography of high contrast surface features that appear in at least two images, as shown in Figure 3. However, many objects commonly measured do not naturally exhibit such features. Traditionally, circular retroreflective targets are attached to the object to artificially provide these high contrast features. When illuminated, with a camera flash for example, these targets reflect light directly back to the illuminating source, causing the targets to appear very bright in relation to the background. Retroreflective targets work exceptionally well and have few drawbacks when used on solid structures ${ }^{8}$. Retroreflective materials are available from a variety of commercial sources; for this experiment the materials selected were provided by $3 \mathrm{M}$ Corporation (St.Paul, Minnesota). To cross-correlate the random XYZ locations of the retroreflective targets with the internal coordinate system of the CM an independent laser scan/survey is performed. The scan provides a global map of all targets in a coordinate system defined by an origin coinciding with the CG as determined by the computer-aided design (CAD) model of the crew module. The CG as determined by the new photogrammetric method will be described by a "delta" to the origin of the coordinate reference.

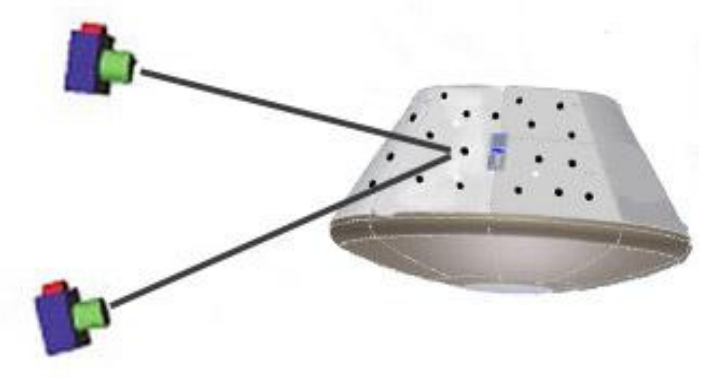

Figure 3. High contrast targets visible from multiple perspectives are required for accurate photogrammetric measurements.

\section{Experimental Approach}

Even though the laboratory environment provides the best setting for any measurement task including photogrammetric measurements it is possible to obtain high resolution/high accuracy position and shape measurements during field test applications if environmental conditions can be managed in such a way as to minimize impact. Here photogrammetry was used to measure the position of the full-scale $17,000 \mathrm{lb}$. CM at different hang angles using an asymmetric two-point lifting strap. The $\mathrm{CM}$ was designed with four lifting points on the top surface (Figure 4). Each lifting point was positioned at a 90-degree offset to the next. Two pairs of lifting straps were provided with designed hang angles of 21 and 30 degrees. Load design limits dictated that the lifting straps be connected only to opposite-side lifting points. The combination of the two straps along with the four lifting points provided eight unique positions from which the CM could be suspended. The diagram in Figure 5 
outlines the configurations of the straps with respect to the fixed lifting points on the crew module and sample images from the configurations.

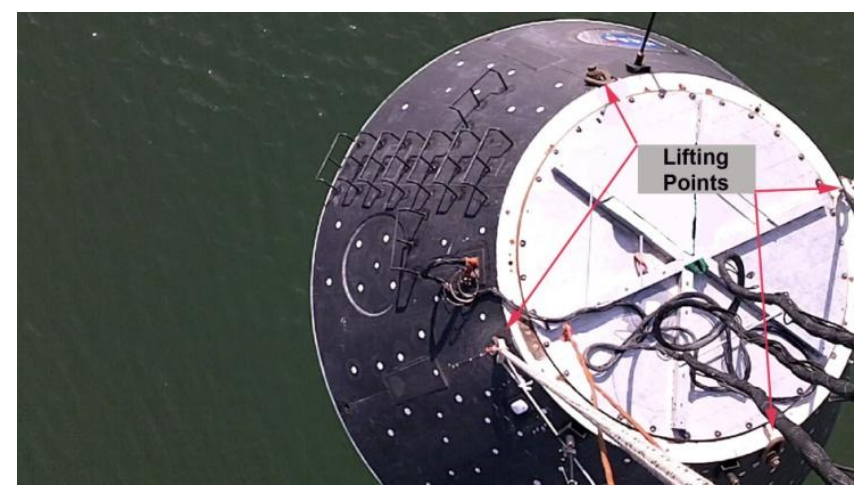

Figure 4. MLAS Crew Module with identified symmetric lifting points

It was theorized that a targeted plumb line suspended from the same lifting hook as the CM would provide an accurate representation of the gravity vector along which the true CG of the vehicle would lie. To measure the position of the plumb line in $3 \mathrm{D}$ space a three-camera photogrammetric network was positioned to achieve the best triangulated solution of the plumb line targets and those on the surface of the CM from different perspectives. The plumb line was constructed with 3-inch diameter retro-reflective spheres positioned along its length (Figure 6a). For each configuration the position of the plumb line(s) and the $\mathrm{CM}$ were recorded and photogrammetrically processed to find the position of the $\mathrm{CM}$ and the plumb line in $3 \mathrm{D}$ space.
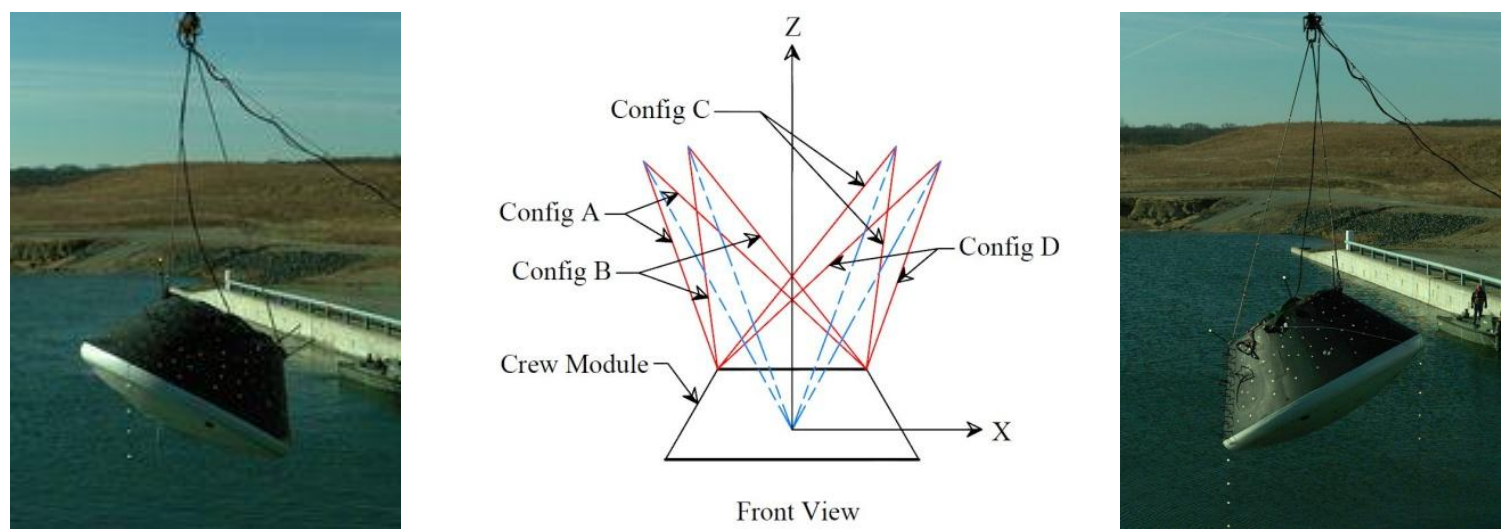

Figure 5. Two of the eight lifting strap configuration(s). The plumb line (not shown) suspended from the same hook projects a line through the $C G$.

Ultimately, this experiment was repeated three times over a period of several months. Each time lessons learned from the previous attempt were implemented. Most notable among the factors that influenced the results was the error introduced by motion and wind. Since the plumb line must be unconstrained on one end, wind is capable of causing small movements. To provide redundancy and quantify this effect, an additional stationary plumb line was included. The additional plumb line with the same type of retroreflective markers was added to the field of view adjacent to crew module, but not attached to the crane. The position of the additional plumb line would be calculated photogrammetrically in the coordinate space defined by the crew module as well. To insure the maximum stability in the second plumb line wind shields were added. The plumb bob itself was submerged in a container of oil but allowed to hang freely to dampen any motion in the plumb line. The second plumb line 
ultimately provided a stable gravitational reference. To correct for the wind effects on the crane line the angle formed between the two plumb lines was calculated photogrammetrically and used to correct the orientation of the line attached to the crane. To further support the accuracy of the method a height-adjustable retroreflective target was added to the lifting ring at the projected intersecting point of the two lifting lines. This vertex point is critical for defining the translation of the plumb line if the experiment were performed without an on-board plumb line attached to the structure. In our experiment it served as a level of redundancy. The target representing the vertex of the intersecting lift lines can be seen in Figure 6.a. To check the position of the lifting ring target additional retroreflective markers were wrapped at two foot intervals along the length of each strap. The virtual line created by these points has a projected intersection at the same position as the target in the lifting ring The point serves as a reference for translating the secondary "stationary" plumb line also visible in Figure 6.b.

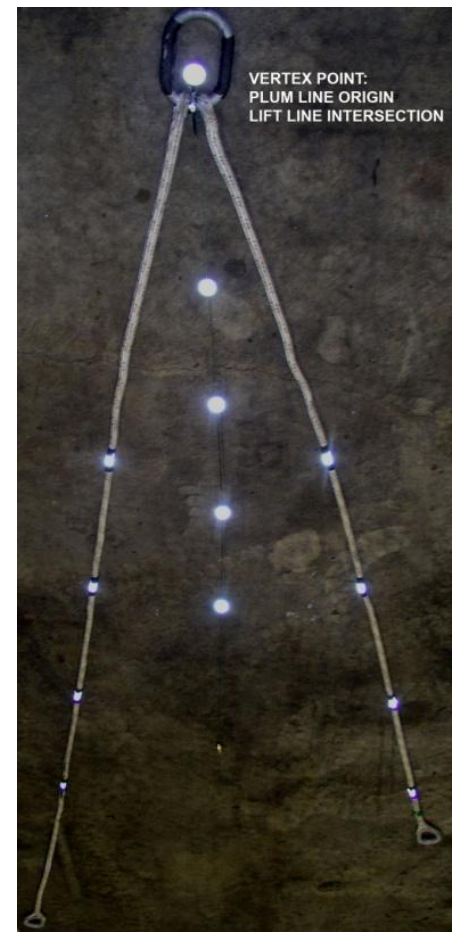

Figure 6.a (Close-up) Lifting strap and plumb line with 3-inch retro-reflective spheres attached.

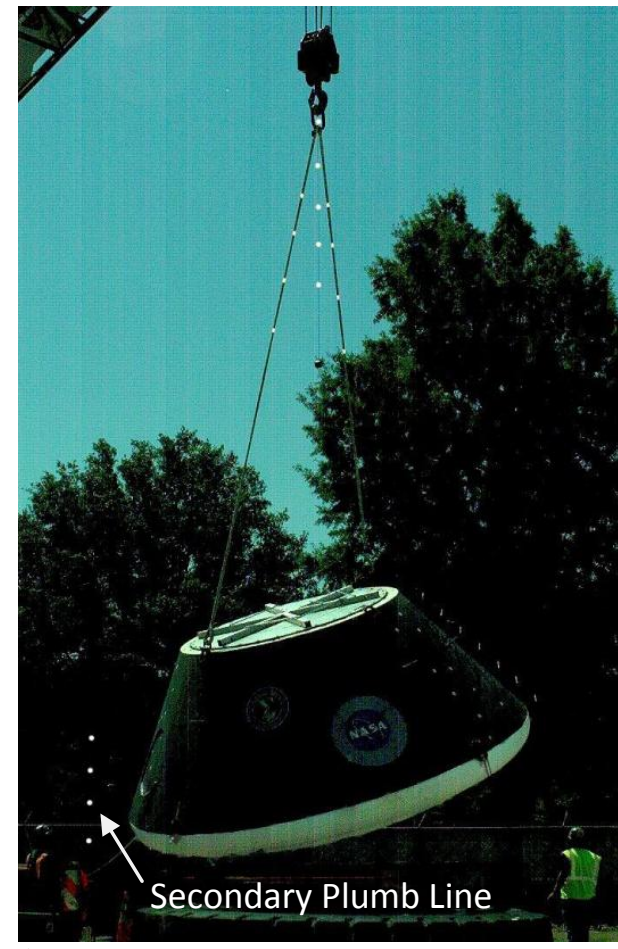

Figure 6.b Typical image from recorded sequence. Stationary plumb line visible to the left of the CM. Retroreflective markers on lifting strap highly visible in daylight conditions.

For this experiment the approximate 23 -foot distance from the reference at the vertex to the CG has the potential to create a large error from small movements. The graphic in Figure 7 depicts the error that may be introduced in the triangulation of the plumb line from the minimum of two positions. Here the intersecting lines are depicted as measured from opposite 30 degree lift positions (Config A and Config $\mathrm{C}$ referenced in Figure 5). In this example with a randomly selected 1.5 degree error which could be attributable to wind or motion, significant errors in the described CG could be expected. 


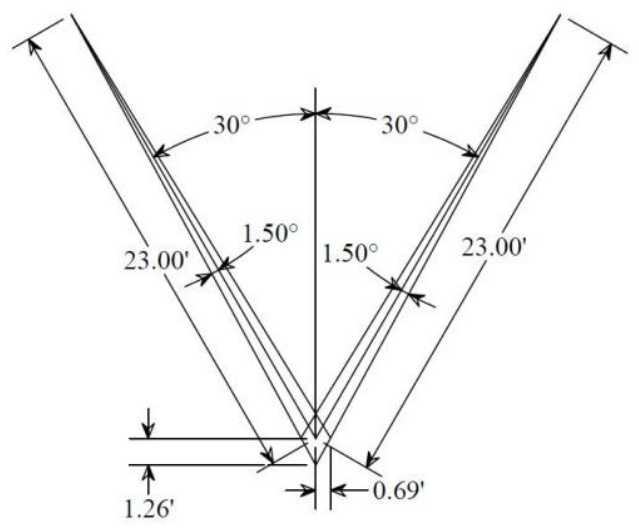

Figure 7. Experimental error potential from factors such as the wind effect on the plumb line.

\section{Image Acquisition and Processing}

A time-synchronized video sequence from three Phantom ${ }^{\mathrm{TM}}$ cameras were recorded at a resolution of $1632 \times 1200$ pixels for a 30 second period. To assist with the motion analysis the cameras were operated at $100 \mathrm{hz}$. The cameras were carefully positioned to ensure each had an unobstructed view of the CM and each of the plumb lines. The images extracted from the video sequence were digitally processed in order to calculate accurate centroid locations of the targets. The resulting measurement data were first evaluated to analyze the best fit line through the points represented by the spheres for both the on-board and stationary plumb lines. Both plumb lines had a relatively low RMS value representing the best fit through the four points, but the stationary plumb line maintained the lowest RMS error of the points for each of the eight configurations. To verify the CM was motionless the recorded video sequence was analyzed to compare the absolute change in position of five randomly selected targets on the CM during a 10 second period of the recorded sequence. The same analysis was performed to verify the stability of the stationary plumb line. The calculated motion (delta change in position) expressed as a RMS over the 10 second sequence for all points was less than 0.014 inches. The "best-fit" solution for the plumb line data is shown in Figure 8. The highlighted data identified as "PlumbLn1_Config" represents the stationary plumb line. The low RMS for PlumbLn1 data suggests wind and/or other factors may have been less than for the on-board plumb line. Four of the measurements (Config 1-4) for the on-board plumb line have been removed from the data set because of inconsistencies in calculation of the line fit. Close examination of the centroid calculation of individual point/target data suggest inconsistencies in the 3D solution for the points when computed by a two or three cameras triangulation suggesting an anomaly likely caused by sun-light or contrast of the target with the background. 


\begin{tabular}{|lrrrrrrrr|}
\hline Name & X & Y & Z & I & J & K & Length & RMS \\
\hline PlumLn1_Config1 & 26.3415 & 38.4236 & -194.6681 & 0.8596 & -0.5107 & 0.0155 & 74.9837 & 0.0030 \\
PlumLn1_Config2 & 81.3772 & 174.6648 & -146.3581 & 0.8627 & 0.0215 & 0.5053 & 74.9757 & 0.0023 \\
PlumLn1_Config3 & -4.3624 & 17.0444 & -165.1730 & 0.8509 & 0.5244 & 0.0316 & 75.0084 & 0.0015 \\
PlumLn1_Config4 & -49.4195 & 71.9178 & -84.5934 & 0.8629 & -0.0015 & -0.5054 & 75.0020 & 0.0004 \\
PlumLn1_Config5 & 33.7130 & 86.9420 & -180.3460 & 0.9401 & -0.3406 & 0.0116 & 72.1811 & 0.2464 \\
PlumLn1_Config6 & 74.0015 & 45.2587 & -200.0545 & 0.9347 & 0.0347 & 0.3536 & 74.9592 & 0.0017 \\
PlumLn1_Config7 & 2.4640 & 2.3366 & -183.6395 & 0.9218 & 0.3872 & 0.0173 & 75.0770 & 0.0030 \\
PlumLn1_Config8 & 17.2981 & 5.1996 & 45.6146 & 0.9345 & 0.0130 & -0.3558 & 73.6045 & 0.1492 \\
\hline PlumLn2_Config5 & 17.6563 & 45.6753 & -1.6424 & 0.9328 & -0.3597 & 0.0244 & 42.2930 & 0.1091 \\
PlumLn2_Config6 & 17.3852 & 0.8493 & -45.5328 & 0.9340 & 0.0151 & 0.3569 & 42.4362 & 0.0460 \\
PlumLn2_Config7 & 16.2203 & -43.9893 & -2.7702 & 0.9382 & 0.3432 & 0.0438 & 42.5982 & 0.0297 \\
PlumLn2_Config8 & 17.2080 & 3.6478 & 45.7892 & 0.9361 & 0.0007 & -0.3518 & 42.8324 & 0.1340
\end{tabular}

Figure 8. Best linear fit thru points on plumb line. Highlighted points represent stationary plumb line; others represent on-board plumb line attached to crane hook. All units are in inches.

To analyze their impact on the CG solution the calculation will be evaluated with all data included for the stationary plumb line and the data from the uncorrected on-board plumb line. The data in Table 1 provides a summary of the angle formed between the two plumb lines. A close inspection of the stationary plumb line prior to each measurement concluded the angle between the two lines was a result of motion on the on-board plumb line. The onboard plumb line was more subjectively determined from a greater distance to not be in motion. For this analysis the stationary plumb line will be translated to the vertex point when used as the gravity reference. Figure (9.a) provides a data visualization of the translated plumb lines and point cloud of the surface targets on the CM.

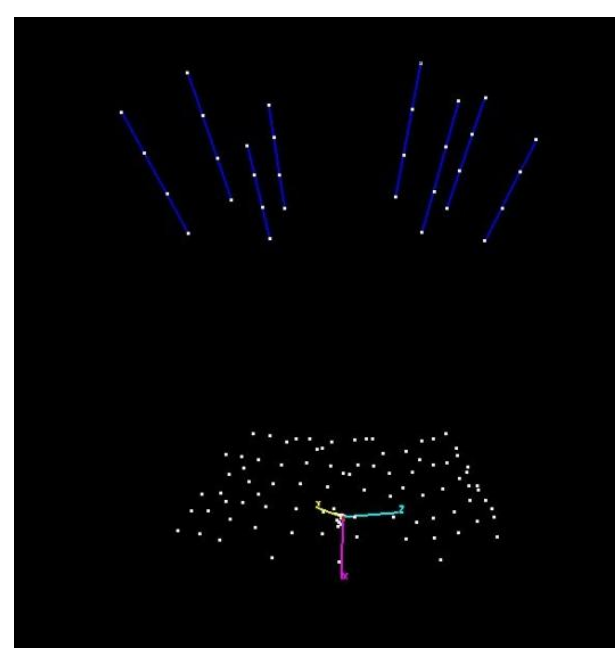

Figure 9.a Compiled plumb line locations (blue) relative to local coordinate system of the crew module.

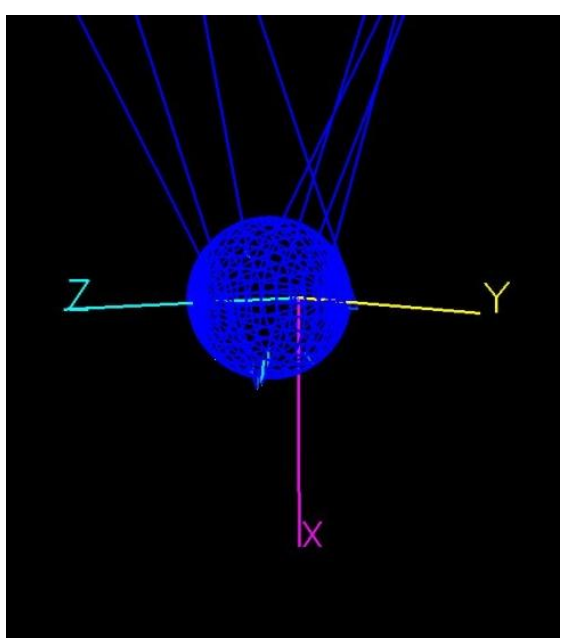

Figure 9.b Magnified view of origin where plumb lines reach closest point of intersection.

A set of custom designed image processing and data reduction functions were developed using the MATLAB ${ }^{\circledR}$ software environment ${ }^{9}$. Supporting functions for image processing, photogrammetry, and coordinate transformations were also implemented in a similar manner. The locations of accurate image plane coordinates (centroids) of targets on the crew module and plumb lines were crucial to the measurement process.

The images were photogrammetrically processed to determine the spatial coordinates of the visible targets on both plumb lines and the crew module ${ }^{10}$. Ideally, the point formed by the intersection of any two of the plumb lines would indicate the crew module CG. However, due to experimental error, the plumb lines do not perfectly intersect. Instead, the plumb lines can be described as intersecting within a spherical region of interest Figure 9.b. To 
determine the center of gravity, a numerical optimization algorithm was implemented that searched the region of interest for a point that minimized the sum of the squared distances from all plumb lines. The purpose was to find a point $\boldsymbol{x}_{0}$ that minimized the objective function shown in Eq. (1). The CG estimate corresponds the point $\boldsymbol{x}_{0}$, that minimizes d, as shown in Eq. (7). The solution utilizing Eq. (2) - (7) was implemented in MATLAB ${ }^{\circledR}$ with the utilization of existing optimization functions.

$$
D=\sum_{i=1}^{N} d_{i}^{2}
$$

where $N$ is the number of plumb lines used in the calculation, and $d$ is the shortest distance between a line defined by two points $\boldsymbol{x}_{1}, \boldsymbol{x}_{2}$ and a point $\boldsymbol{x}_{0}$, as shown in Figure 10. In three dimensions the line is represented by points $x_{1}=\left(\mathrm{x}_{1}, \mathrm{y}_{1}, \mathrm{z}_{1}\right)$ and $x_{2}=\left(\mathrm{x}_{2}, \mathrm{y}_{2}, \mathrm{z}_{2}\right)$

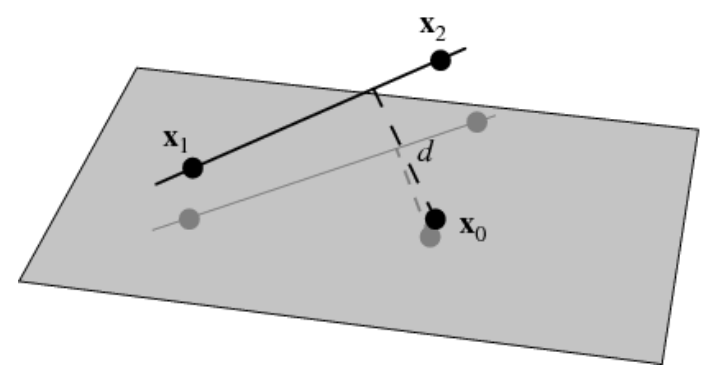

Figure 10. Depicted point to line distance in 3-dimensional space $\left(x_{0}\right)$ represents the CG

The squared distance between a point on the line with parameter $t$ and a point $\boldsymbol{x}_{0}$ is

$$
\begin{gathered}
d^{2}=\left[\left\langle x_{1}-x_{0}\right\rangle+\left\langle x_{2}-x_{1}\right\rangle t\right]^{2}+\left[\left\langle y_{1}-y_{0}\right\rangle+\left\langle y_{2}-y_{1}\right\rangle t\right]^{2} \\
+\left[\left\langle z_{1}-z_{0}\right\rangle+\left\langle z_{2}-z_{1}\right\rangle t\right]^{2}
\end{gathered}
$$

Solving for $\mathrm{t}$; with the use of the dot product gives

$$
t=-\frac{\left\langle x_{1}-x_{0}\right\rangle \cdot\left\langle x_{2}-x_{1}\right\rangle}{\left|x_{2}-x_{1}\right|^{2}}
$$

Plugging t back into Eq. (1) yields

$$
\begin{aligned}
d^{2}=\left\langle x_{1}-x_{0}\right\rangle^{2} & +\left\langle y_{1}-y_{0}\right\rangle^{2}+\left\langle z_{1}-z_{0}\right\rangle^{2} \\
& +2 t\left[\left\langle x_{2}-x_{1}\right\rangle\left\langle x_{1}-x_{0}\right\rangle+\left\langle y_{2}-y_{1}\right\rangle\left\langle y_{1}-y_{0}\right\rangle+\left\langle z_{2}-z_{1}\right\rangle\left\langle z_{1}-z_{0}\right\rangle\right] \\
& +t^{2}\left[\left\langle x_{2}-x_{1}\right\rangle^{2}+\left\langle y_{2}-y_{1}\right\rangle^{2}+\left\langle z_{2}-z_{1}\right\rangle^{2}\right]
\end{aligned}
$$

Which can be reduced to

$$
=\frac{\left|x_{1}-x_{0}\right|^{2}\left|x_{2}-x_{1}\right|^{2}-\left[\left\langle x_{1}-x_{0}\right\rangle \cdot\left\langle x_{2}-x_{1}\right\rangle\right]^{2}}{\left|x_{2}-x_{1}\right|^{2}}
$$


Applying the vector quadruple product (Eq. 6) to (Eq. 5), where $\mathrm{x}$ denotes the cross product.

$$
\begin{aligned}
& (A \times B)^{2}=A^{2} B^{2}-(A \cdot B)^{2} \\
& d=\frac{\left|\left(\boldsymbol{x}_{2}-\boldsymbol{x}_{1}\right) \times\left(\boldsymbol{x}_{1}-\boldsymbol{x}_{0}\right)\right|}{\left|\left(\boldsymbol{x}_{2}-\boldsymbol{x}_{1}\right)\right|}
\end{aligned}
$$

Here the numerator is twice the area of the triangle formed by the points $\boldsymbol{x}_{\boldsymbol{\theta}}, \boldsymbol{x}_{1}$, and $\boldsymbol{x}_{2}$ and the denominator is the length of one of the bases of the triangle ${ }^{11}$. Equations (2) - (7) were formulated from discussions found in Reference 11.

The computed CG suggests that some of the measurements were likely influenced by the conditions at the test site. Data comparisons were made using uncorrected plumb line data from the on-board plumb line to that from the stationary plumb line. The on-board data excluded plumb lines from configurations (1-4) that were thought to be excessively influenced by wind or motion. For comparison the on-board plumb line data was uncorrected for the angle between it and the stationary plumb line to highlight the effects of a small offset. The angle between the two groups is described for each configuration in Table 1.

Table 1. Angle between on-board plumb line and stationary plumb line.

\begin{tabular}{ll} 
Line to Line & Angle \\
\hline XPlumLn1_Config5 to PlumLn2_Config5 & 1.3860 \\
XPlumLn1_Config6 to PlumLn2_Config6 & 1.1421 \\
XPlumLn1_Config7 to PlumLn2_Config7 & 1.4950 \\
XPlumLn1_Config8 to PlumLn2_Config8 & 0.7499
\end{tabular}

These results show the stationary plumb line yielded a smaller offset to the original CG (CAD estimated). The results determined by this method are shown as an offset from the origin (Table 2). As expected the data from the stationary plumb line provides the closest match to the original estimate, and suggests the largest shift occurs in the $\mathrm{x}$-axis. This can be potentially explained by the removal of avionics hardware that was included in the original estimate, but removed prior to our testing. The hardware removed was estimated at $1 \%$ of total $\mathrm{CM}$ weight.

Table 2. Comparison of Photogrammetric CG solution: Using "stationary" Plumb Line Data vs. Selected "onboard" Plumb Line Data (wind points removed). Data expressed as an offset from CAD defined CG

\begin{tabular}{|l|l|}
\hline \multicolumn{2}{|c|}{ CG Determination } \\
\hline $\begin{array}{c}\text { CG computed using data from "uncorrected" on- } \\
\text { board plumb line }\end{array}$ & $\begin{array}{c}\text { CG computed using "stationary" plumb line data } \\
\text { from 8 configurations }\end{array}$ \\
\hline X: -0.807 (inches) & X: -1.40 (inches) \\
\hline Y: 1.372 & Y: 0.372 \\
\hline Z: -1.749 & Z: -0.561 \\
\hline
\end{tabular}




\section{Conclusions}

Using the lessons learned through this experiment it is concluded this CG determination technique can provide reliably accurate results suitable for most applications. The analysis was performed with a careful application of photogrammetric principles and tools to achieve the best results. During the execution of this experiment a greater understanding of the factors that contribute to the overall error was gained. This method eliminated the need for costly and complex support equipment that would have been required for CG determination via traditional methods in a laboratory setting. The technique lends itself well to large bodies, oddly-shaped bodies, and fragile flight hardware and it can be executed more quickly than traditional measurement techniques. It is generally recommended that the technique be performed within an enclosed area, this would eliminate the issues associated with wind induced motion and glare from the sun discussed in the analysis. The suitability of this technique should be considered on a case to case basis where the geometry of the lift lines and the test article are considered along with recognition that potential motion of the plumb line or the test article could introduce significant error with very small motions. The preferred method would be the use of a "stationary" plumb line to insure minimal effects from external factors such as wind and vibration. If this approach is chosen the need for an accurately placed target at the vertex of the lifting lines is required. It is recommended the lifting straps be considered as a potential source of friction and analyzed for their influence, for this case the effects of friction at the connection points was considered insignificant due to the weight of the $\mathrm{CM}$. The technique presented here would appear to be suitable when combined with well-documented but rarely-used pendulum methods capable of measuring other flight hardware physical properties including moments of inertia (MOI's) ${ }^{12}$. Additional experimental testing is being conducted to determine the adaptability of the technique for MOI type measurement.

\section{Acknowledgements}

The authors thankfully recognize the efforts and significant contributions made during the planning, development, and implementation of the photogrammetric measurements for CG determination. Acknowledgement is made to the NASA Engineering and Safety Center (NESC) for their continued support during the development and refinement of this CG measurement technique. Further acknowledgement is extended to John Baker (NASA JPL) and Michael

Kelly (NASA NESC) for program leadership along with the entire Crew Module Water Landing Model test team for their strong support of this effort. In addition Kurt Severance (NASA LaRC) is acknowledged for technical support and contributions in many areas of data visualizations and analysis. Dan Yuchnovicz (NASA NESC) is acknowledged for consultation on design and implementation of this experimental technique. Further acknowledgement is extended to Paul Bagby (NASA LaRC) for operational support of the video system hardware. 


\section{References}

1 Fregolent, A., Sestieri, A., "Identification of Rigid Body Inertia Properties from Experimental Data" Mechanical Systems and Signal Processing, 1996.

2 Kloepper, R, Akita, H., Okuma, M. "An Experimental Identification Method for Rigid Body Properties Enabled by GravityDependent Suspension Modeling" The 1st joint international conference on Multibody System Dynamic, Finland, May 2010.

3 Almeida, R.A.B., Urgueira, A.P.V, et al., "Further Developments on the Estimation of Rigid Body Properties from Experimental Data" Elsevier, Mechanical Systems and Signal Processing, 2010.

4 Brewer, Willima V., "Space Station racks weight and CG measurement using the rack insertion end-effector", Univ. of Central Florida, NASA/ASEE Summer Faculty Fellowship Prog. 1994 Research Reports p 31-60 (SEE N95-18166)

5 Schedlinksi, C., Link, M., "A Survey of Current Inertia Parameter Identification Methods" Mechanical Systems and Signal Processing, 2001, issue:15, pg 189-211

6 Luhmann,T., Robson,S., Kyle,S.,A., Harley,I.,A, Close Range Photogrammetry: Principles, Techniques and Applications, John Wiley \& Sons, 2006. ISBN: 1-870325-50-8.

7 Mikhail, E.M., Bethel, J.S., and McGlone, J.C., Introduction to Modern Photogrammetry, John Wiley \& Sons, pp. 124-125, 2001.

8 Clarke, T.A., "An analysis of the properties of targets used in digital close range phtogrammetric measurement", SPIE: Videometrics III, Boston, Massachusetts. November 1994,

9 Liu, T. and Burner, A.W., Photogrammetry Toolbox User's Guide, Western Michigan University, Kalamazoo, Michigan, May 2007.

10 Jones, Thomas W., et. Al., "Photogrammetric Measurement Methods", Recent Advances in Gossamer Spacecraft, C.H.M. Jenkins (Editor), Vol. 212, Progress in Astronautics and Aeronautics Series, Published by AIAA, @ 2006, 344 pages, Hardback ISBN-10: 1-56347-777-7.

11 Weisstein, E.W. "Point-Line Distance-3-Dimensional.” MathWorld- A Wolfram Web Resource. http://mathworld.wolfram.com/Point-LineDistance3-Dimensional.html.

12 Wolowicz, Chester H. and Yancey, Roxanah B., 1974, "Experimental determination of airplane mass and inertial characteristics". NASA Technical Report TR R-433. 